\title{
Stability Analysis of a Car-Following Model on Two Lanes
}

\author{
Yuhan Jia, ${ }^{1,2}$ Yiman Du, ${ }^{1,2}$ and Jianping $W u^{1,2}$ \\ ${ }^{1}$ Department of Civil Engineering, Tsinghua University, Beijing 100084, China \\ ${ }^{2}$ Jiangsu Province Collaborative Innovation Center of Modern Urban Traffic Technologies, Nanjing 210096, China \\ Correspondence should be addressed to Yiman Du; ymducp@gmail.com
}

Received 16 October 2014; Revised 20 November 2014; Accepted 20 November 2014; Published 17 December 2014

Academic Editor: Sergio Preidikman

Copyright (C) 2014 Yuhan Jia et al. This is an open access article distributed under the Creative Commons Attribution License, which permits unrestricted use, distribution, and reproduction in any medium, provided the original work is properly cited.

Considering lateral influence from adjacent lane, an improved car-following model is developed in this paper. Then linear and nonlinear stability analyses are carried out. The modified Korteweg-de Vries (MKdV) equation is derived with the kink-antikink soliton solution. Numerical simulations are implemented and the result shows good consistency with theoretical study.

\section{Introduction}

Traffic flow is a system of consecutive vehicles with interaction [1]. Recently various models have been developed, including general models, safety distance models, action point models, optimal velocity models (OVM), cellular automaton models, and fuzzy logic models [2-4]. Among those models, OVM developed by Bando et al. $[5,6]$ is well known for its accuracy and rationality. Afterwards Helbing and Tilch [7] calibrated the OV model by experimental data and developed a generalized force model (GFM) to overcome the deficiencies. But both OVM and GFM cannot describe the phenomenon that the following vehicle may not decelerate when the leading vehicle is much faster even if the headway distance is smaller than safety distance. Then the inconsistencies of previous models were overcome by a continuous microscopic single-lane model, the intelligent driver model, developed by Treiber et al. [8] by the analysis of German freeways data. After this, a full velocity difference model (FVDM) was developed by Jiang et al. $[9,10]$ to solve the disadvantage. However, there are still some problems in previous models, which are discussed in detail and improved by Treiber and Kesting [11]. Also the gas-kinetic-based model was investigated by observed data and simulation experiments, which showed good agreement with phenomena in reality [12]. In this century, many new models have been established by considering decentralized delayed-feedback control [13], delay time due to driver's reaction [14], extended
OV function for acceleration difference [15], multiple velocity difference [16], and optimal velocity difference [17].

To study traffic jam waves in OVM, Komatsu and Sasa [18] firstly derived the modified Korteweg-de Vries (MKdV) equation to describe kink waves. Then Muramatsu and Nagatani [19] derived Korteweg-de Vries (KdV) equation from OVM to describe sliton waves in traffic jam, and Nagatani also found triangular shock wave solved Burgers equation [20]. From then many models have been analyzed by nonlinear stability theory aforementioned. Nagatani [21] derived MKdV equation near critical point in two continuum models: partial differential and discrete lattice model. Yu [22] presented a simplified OVM considering relative velocity and derived $\mathrm{KdV}$ and $\mathrm{MKdV}$ equations. Ge et al. developed several intelligent transportation system (ITS) based models with $\mathrm{KdV}$ and MKdV analysis [23] and also did similar research in three OVM based models [24]. Yu et al. [25] recently build a two-delay model with $\mathrm{MKdV}$ investigation and implemented numerical simulations. More studies show that the triangular wave, soliton wave, and kink wave occur in stable region, metastable region, and unstable region, respectively [20, 26, 27].

However, only a few researches focused on car-following with lateral impact, in which case the lateral influence from adjacent lane should be considered. Nagatani [28] presented two lattice models to simulate traffic flow wave on a twolane highway with lane changing. Jin et al. [29] considered the lane-width influence and developed a non-lane-based 
FVDM with simulation experiments. Ge et al. [30] studied the influence from neighbor vehicle or nonmotor vehicle by considering two more OV functions and analyzed the stability condition by control theory method. Based on previous work, this paper investigates a new car-following model considering lateral influence by introducing the combination of two OV functions. In Section 2 the new model is developed and linear stability analysis is carried out in Section 3. In Section 4 the MKdV equation is derived to obtain kink-antikink soliton solution. Then numerical simulation experiments are performed to verify the theoretical study in Section 5. The summary is given in Section 6.

\section{Improved OVM}

The typical OV model is presented as $[5,6]$

$$
\frac{d^{2} x_{n}(t)}{d t^{2}}=\alpha\left[V^{\mathrm{op}}\left(\Delta x_{n}(t)\right)-v_{n}(t)\right]
$$

where $x_{n}(t)$ and $v_{n}(t)$ are the position and velocity of the $n$th vehicle, $\Delta x_{n}(t)$ is the headway distance between the $n$th and its leading vehicle, $\alpha$ is the sensitivity parameter of the driver, and $V^{\mathrm{op}}(\cdot)$ is the optimal velocity function described as [5]

$$
V^{\mathrm{op}}\left(\Delta x_{n}(t)\right)=\frac{v_{\max }}{2}\left[\tanh \left(\Delta x_{n}(t)-h_{c}\right)+\tanh \left(h_{c}\right)\right],
$$

where $v_{\max }$ is the maximum velocity on a particular roadway and $h_{c}$ means the safety headway distance.

However, as noticed in the study on roadway, a driver usually focuses not only the leading vehicle on the present lane, but also the vehicle on adjacent lane, especially when the neighbor vehicle decelerates. This phenomenon occurs because of the potential action of lane changing or the avoidance of collision when the lane width is small [29]. Hence the lateral influence should be considered in carfollowing model even if lane changing does not occur.

It is assumed that the driver makes his decision upon the combination impact of leading vehicle and neighbor vehicle by introducing a second OV function, which can be defined as

$$
\bar{V}^{\mathrm{op}}\left(l_{n}(t)\right)= \begin{cases}V^{\mathrm{op}}\left(\Delta x_{l, n+1}(t)\right), & l_{v} \leq \Delta x_{l, n+1}(t)<d \\ 0, & \text { others }\end{cases}
$$

where $\Delta x_{l, n+1}(t)$ is the headway distance between the $n$th vehicle and its leading vehicle on the adjacent lane, $l_{v}$ is the length of a normal vehicle, and $d$ is a preset constant.

Referring to previous study, $\Delta v_{n}(t)$ and $\Delta v_{l, n}(t)$ are introduced [7], where $\Delta v_{n}(t)=v_{n+1}(t)-v_{n}(t)$ and $\Delta v_{l, n}(t)$ can be given as

$$
\Delta v_{l, n}(t)= \begin{cases}v_{l, n}(t)-v_{n}(t), & l_{v} \leq \Delta x_{l, n+1}(t)<d, \\ 0, & \text { others, }\end{cases}
$$

in which $v_{l, n}(t)$ is the velocity of the leading vehicle on adjacent lane.
The new model can be expressed as

$$
\begin{aligned}
\frac{d^{2} x_{n}(t)}{d t^{2}}= & \alpha\left[p V^{\mathrm{op}}\left(\Delta x_{n}(t)\right)+q \bar{V}^{\mathrm{op}}\left(\Delta x_{l, n}(t)\right)-v_{n}(t)\right] \\
& +\lambda_{1} \Delta v_{n}(t)+\lambda_{2} \Delta v_{l, n}(t)
\end{aligned}
$$

where $p$ and $q$ are the weights of the two OV functions and $\lambda_{1}$ and $\lambda_{2}$ are the weights of velocity difference.

\section{Linear Stability Analysis}

According to linear stability analysis method [6], stable condition of the uniform traffic flow is given by

$$
\begin{aligned}
& x_{n}^{(0)}(t)=n h_{c}+V^{\mathrm{op}}(h) t, \\
& x_{l, n}^{(0)}(t)=n h_{c}+\bar{V}^{\mathrm{op}}(h) t .
\end{aligned}
$$

Let $y_{n}(t)$ and $y_{l, n}(t)$ be small deviations from $x_{n}^{(0)}(t)$ and $x_{l, n}^{(0)}(t)$ as $x_{n}(t)=x^{(0)}(t)+y_{n}(t)$ and $x_{l, n}(t)=x_{l}^{(0)}(t)+y_{l, n}(t)$. The linearized equation can be obtained:

$$
\begin{aligned}
\frac{d^{2} y_{n}(t)}{d t^{2}}= & \alpha\left[p V^{\prime}\left(\Delta x^{(0)}\right) \Delta y(t)\right. \\
& \left.+q \bar{V}^{\prime}\left(\Delta x_{l}^{(0)}\right) \Delta y_{l}(t)-v_{n}(t)\right] \\
& +\lambda_{1} \Delta v_{n}(t)+\lambda_{2} \Delta v_{l n}(t),
\end{aligned}
$$

where $V^{\prime}$ and $\bar{V}^{\prime}$ are the derivatives of OV functions $V^{\mathrm{op}}\left(\Delta x_{n}(t)\right)$ and $\bar{V}^{\mathrm{op}}\left(l_{n}(t)\right)$. By expanding $y_{n}(t) \propto \exp (i k n+$ $z t)$ and $y_{l, n}(t) \propto \exp (i k n+z t),(7)$ can be rewritten as

$$
\begin{aligned}
& z^{2}+\left[\alpha-\left(\lambda_{1}+\lambda_{2}\right)\left(e^{i k}-1\right)\right] z \\
& \quad-\alpha\left(p V^{\prime}+q \bar{V}^{\prime}\right)\left(e^{i k}-1\right)=0 .
\end{aligned}
$$

Then expand $z$ by the order of $i k$ at the point of $i k \approx 0$ as $z=z_{1} i k+z_{2}(i k)^{2}+\cdots$ and insert it into (8). The following terms can be obtained:

$$
\begin{gathered}
z_{1}=p V^{\prime}+q \bar{V}^{\prime} \\
z_{2}=\left(\frac{1}{2}+\frac{\lambda_{1}+\lambda_{2}}{\alpha}\right)\left(p V^{\prime}+q \bar{V}^{\prime}\right)-\frac{\left(p V^{\prime}+q \bar{V}^{\prime}\right)^{2}}{\alpha} .
\end{gathered}
$$

According to previous study, the vehicle system is stable when $z_{2}>0$, which is

$$
\alpha>2\left(p V^{\prime}+q \bar{V}^{\prime}\right)-2\left(\lambda_{1}+\lambda_{2}\right),
$$

and the neutral stability condition has the following form:

$$
\alpha_{c}=2\left(p V^{\prime}+q \bar{V}^{\prime}\right)-2\left(\lambda_{1}+\lambda_{2}\right)
$$

The stability surface is described in Figure 1. Parameters are set as $v_{\max }=4, h_{c}=7, p=1, q=0, \lambda_{1}=0.2$, and $\lambda_{2}=0$ in Figure 1(a), while $p=0.8, q=0.2, \lambda_{1}=0.16$, and $\lambda_{2}=0.04$ in Figure $1(\mathrm{c})$. As can be seen, the unstable region is smaller considering influence from adjacent lane [25]. 


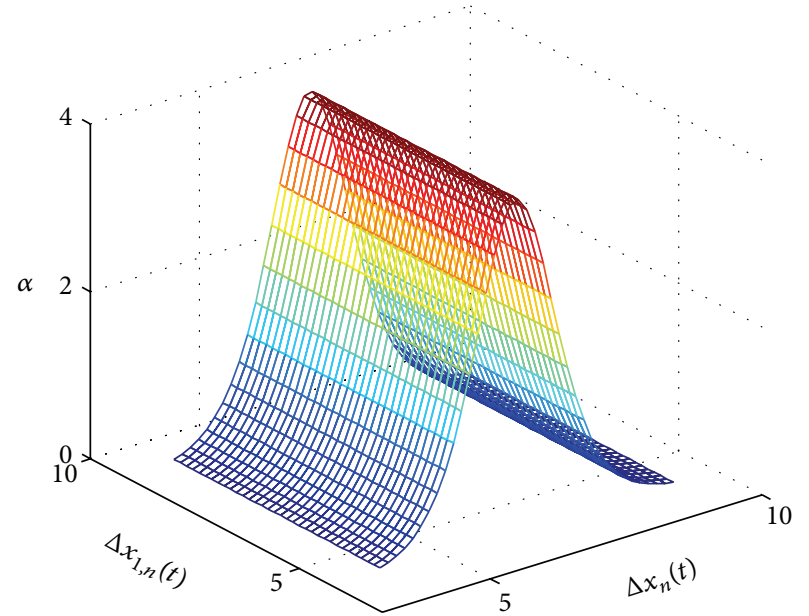

(a)

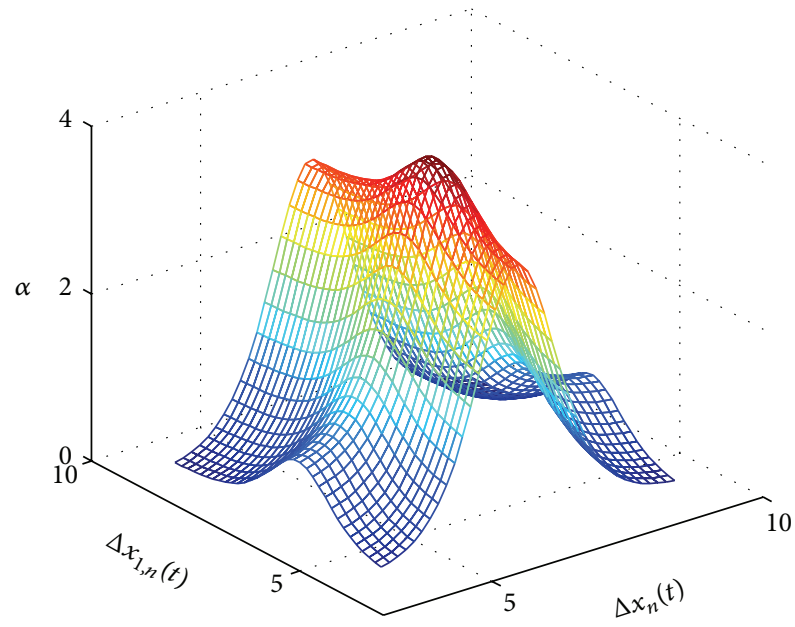

(c)

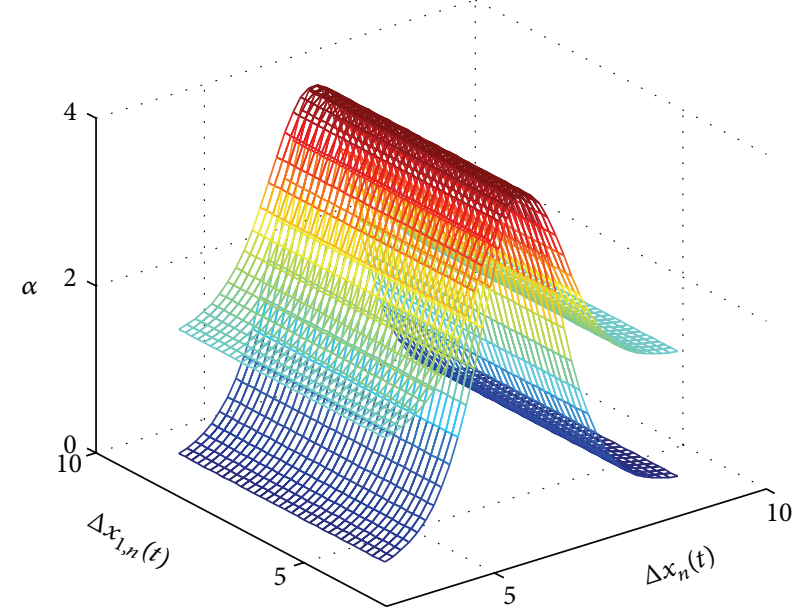

(b)

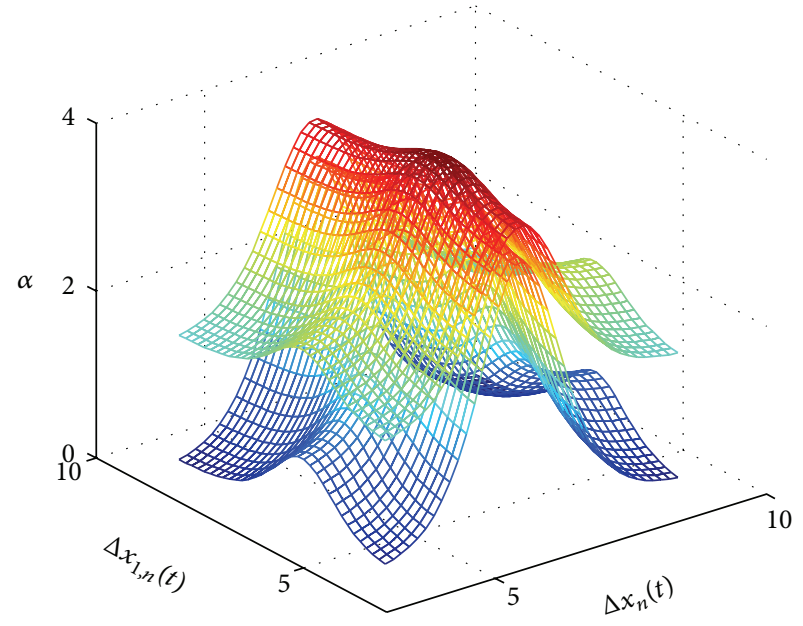

(d)

Figure 1: Headway-sensitivity space for (a) and (b): $p=1, q=0, \lambda_{1}=0.2$, and $\lambda_{2}=0$ and (c) and (d): $p=0.8, q=0.2, \lambda_{1}=0.16$, and $\lambda_{2}=0.04$.

\section{Nonlinear Stability Analysis}

For the convenience of nonlinear analysis, (5) is rewritten as

$$
\begin{aligned}
\frac{d^{2} \Delta x_{n}(t)}{d t^{2}}=\alpha[ & p V\left(\Delta x_{n+1}(t)\right)-p V\left(\Delta x_{n}(t)\right) \\
& +q \bar{V}\left(\Delta x_{l, n+1}(t)\right) \\
& \left.-q \bar{V}\left(\Delta x_{l, n}(t)\right)-\frac{d \Delta x_{n}(t)}{d t}\right] \\
+ & \lambda_{1} \frac{d \Delta x_{n+1}(t)}{d t}-\lambda_{1} \frac{d \Delta x_{n}(t)}{d t} \\
& +\lambda_{2} \frac{d \Delta x_{l, n+1}(t)}{d t}-\lambda_{2} \frac{d \Delta x_{l, n}(t)}{d t}
\end{aligned}
$$

$\mathrm{MKdV}$ equation is obtained in unstable region around the critical point $\left(h_{c}, \alpha_{c}\right)$, where $V^{\prime \prime}=0$. By the analysis method in [18], the long wave expansion is applied in this section.
Two slow scales for space variable $n$ and time variable $t$ are introduced. We define slow variables $X$ and $T$ as

$$
X=\varepsilon(n+b t), \quad T=\varepsilon^{3} t,
$$

where $b$ is a constant determined later and $\varepsilon=\sqrt{\alpha_{c} / \alpha-1}$.

Headways for two lanes are set as

$$
\begin{gathered}
\Delta x_{n}(t)=h_{c}+\varepsilon R(X, T), \\
\Delta x_{l, n}(t)=h_{c}+\varepsilon R(X, T) .
\end{gathered}
$$

Expanding (12) to the fifth order of $\varepsilon$ then gives

$$
\begin{aligned}
& \varepsilon^{2}\left[\alpha b-\alpha\left(p V^{\prime}+q \bar{V}^{\prime}\right)\right] \partial_{X} R \\
& \quad+\varepsilon^{3}\left[b^{2}-\frac{\alpha\left(p V^{\prime}+q \bar{V}^{\prime}\right)}{2}-\left(\lambda_{1}+\lambda_{2}\right) b\right] \partial_{X}^{2} R
\end{aligned}
$$




$$
\begin{aligned}
& +\varepsilon^{4}\left[\alpha \partial_{T} R-\frac{\alpha\left(p V^{\prime \prime \prime}+q \bar{V}^{\prime \prime \prime}\right)}{6} \partial_{X} R^{3}\right. \\
& \left.-\frac{\alpha\left(p V^{\prime}+q \bar{V}^{\prime}\right)+3 b\left(\lambda_{1}+\lambda_{2}\right)}{6} \partial_{X}^{3} R\right] \\
& +\varepsilon^{5}\left[\left(2 b-\lambda_{1}-\lambda_{2}\right) \partial_{X} \partial_{T} R\right. \\
& -\frac{\alpha\left(p V^{\prime}+q \bar{V}^{\prime}\right)+4 b\left(\lambda_{1}+\lambda_{2}\right)}{24} \partial_{X}^{4} R \\
& \left.-\frac{\alpha\left(p V^{\prime \prime \prime}+q \bar{V}^{\prime \prime \prime}\right)}{12} \partial_{X}^{2} R^{3}\right]=0 .
\end{aligned}
$$

It is noticed that the $\partial_{X} \partial_{T} R$ in the sixth order term of (15) can be eliminated by taking the derivative of $X$ in the fifth order term. Then insert $b=p V^{\prime}+q \bar{V}^{\prime}$ and $\alpha_{c} / \alpha-1=\varepsilon^{2}$ into (15); that is,

$$
\begin{aligned}
& {\left[\partial_{T} R-m_{1} \partial_{X}^{3} R+m_{2} \partial_{X} R^{3}\right]} \\
& \quad+\varepsilon\left[m_{3} \partial_{X}^{2} R+m_{4} \partial_{X}^{4} R+m_{5} \partial_{X}^{2} R^{3}\right]=0,
\end{aligned}
$$

where

$$
\begin{aligned}
& m_{1}=\frac{\left(\alpha+3 \lambda_{1}+3 \lambda_{2}\right)\left(p V^{\prime}+q \bar{V}^{\prime}\right)}{6 \alpha}, \\
& m_{2}=-\frac{\left(p V^{\prime \prime \prime}+q \bar{V}^{\prime \prime \prime}\right)}{6}, \\
& m_{3}=\frac{\left(p V^{\prime}+q \bar{V}^{\prime}\right)}{2}, \\
& m_{4}=\left[\frac{4\left(2 p V^{\prime}+2 q \bar{V}^{\prime}-\lambda_{1}-\lambda_{2}\right)\left(\alpha+3 \lambda_{1}+3 \lambda_{2}\right)}{24 \alpha^{2}}\right. \\
& \left.-\frac{\alpha+4\left(\lambda_{1}+\lambda_{2}\right)}{24 \alpha}\right]\left(p V^{\prime}+q \bar{V}^{\prime}\right) \\
& m_{5}=\frac{2\left(2 p V^{\prime}+2 q \bar{V}^{\prime}-\lambda_{1}-\lambda_{2}\right)-\alpha}{12 \alpha}\left(p V^{\prime \prime \prime}+q \bar{V}^{\prime \prime \prime}\right) \text {. }
\end{aligned}
$$

In order to have standard MKdV equation, the following transformations are made in (16):

$$
T=\frac{1}{m_{1}} T_{m}, \quad R=\sqrt{\frac{m_{1}}{m_{2}}} R_{m} .
$$

Then (16) can be rewritten as

$$
\begin{aligned}
& {\left[\partial_{T_{m}} R_{m}-\partial_{X}^{3} R_{m}+\partial_{X} R_{m}^{3}\right]} \\
& +\frac{\varepsilon}{m_{1}}\left[m_{3} \partial_{X}^{2} R_{m}+m_{4} \partial_{X}^{4} R_{m}+\frac{m_{1} m_{5}}{m_{2}} \partial_{X}^{2} R_{m}^{3}\right]=0 .
\end{aligned}
$$

Ignoring the $O(\varepsilon)$ term, we have MKdV equation with a kink-antikink soliton solution expressed as

$$
R_{m 0}\left(X, T_{m}\right)=\sqrt{B} \tanh \left[\sqrt{\frac{B}{2}}\left(X-B T_{m}\right)\right] .
$$

To determine the value of amplitude $B$, the solvable condition is considered:

$$
\left(R_{m 0}, M\left[R_{m 0}\right]\right) \equiv \int_{-\infty}^{\infty} R_{m 0} M\left[R_{m 0}\right] d X=0,
$$

where $M\left[R_{m 0}\right]$ means the $O(\varepsilon)$ term in (19). By performing the integration of (21), the value of amplitude $B$ can be obtained:

$$
B=\frac{5 m_{2} m_{3}}{2 m_{2} m_{4}-3 m_{1} m_{5}} .
$$

The kink-antikink soliton solution of headway can be written as follows:

$$
\begin{aligned}
\Delta x_{n}=h_{c}+\sqrt{\left(\frac{\alpha_{c}}{\alpha}-1\right)\left(\frac{5 m_{1} m_{3}}{2 m_{2} m_{4}-3 m_{1} m_{5}}\right)} \\
\times \tanh \left\{\sqrt{\left(\frac{\alpha_{c}}{\alpha}-1\right) \frac{5 m_{2} m_{3}}{4 m_{2} m_{4}-6 m_{1} m_{5}}}\right. \\
\times\left[n+\left(p V^{\prime}+q \bar{V}^{\prime}\right) t\right. \\
\left.\left.-\left(\frac{\alpha_{c}}{\alpha}-1\right) \frac{5 m_{2} m_{3} t}{2 m_{2} m_{4}-3 m_{1} m_{5}}\right]\right\} .
\end{aligned}
$$

With the amplitude of (23), we have the coexisting surface in Figures 1(b) and 1(d) based on Figures 1(a) and 1(c), respectively. The space is divided into three regions: stable region above the coexisting surface, metastable region between coexisting surface and stability surface, and unstable region below stability surface. However, the limitation should be noted that this nonlinear analysis is only valid at the critical point when $h=h_{c}$.

\section{Numerical Simulation}

Consider a two-lane system with 100 vehicles running on each lane under a periodic boundary condition without overtaking or lane changing. The initial values are $v_{\max }=4$, $h_{c}=7$, and $d=10$ on both lanes. In this section, only the situation of $p+q=1$ is simulated, because we suppose that the sum of OV functions' weights should remain as 1 , which 


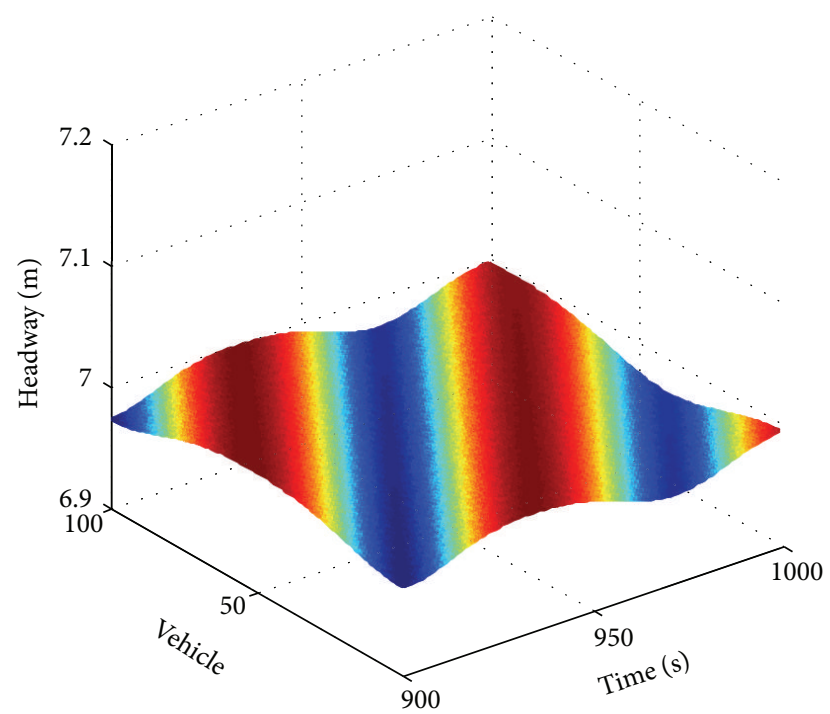

(a)

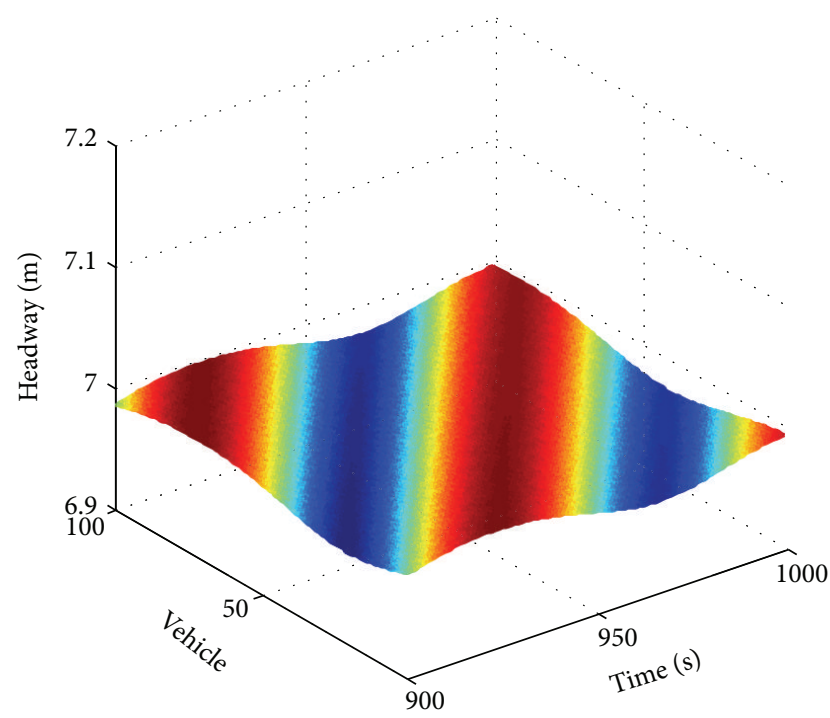

(c)

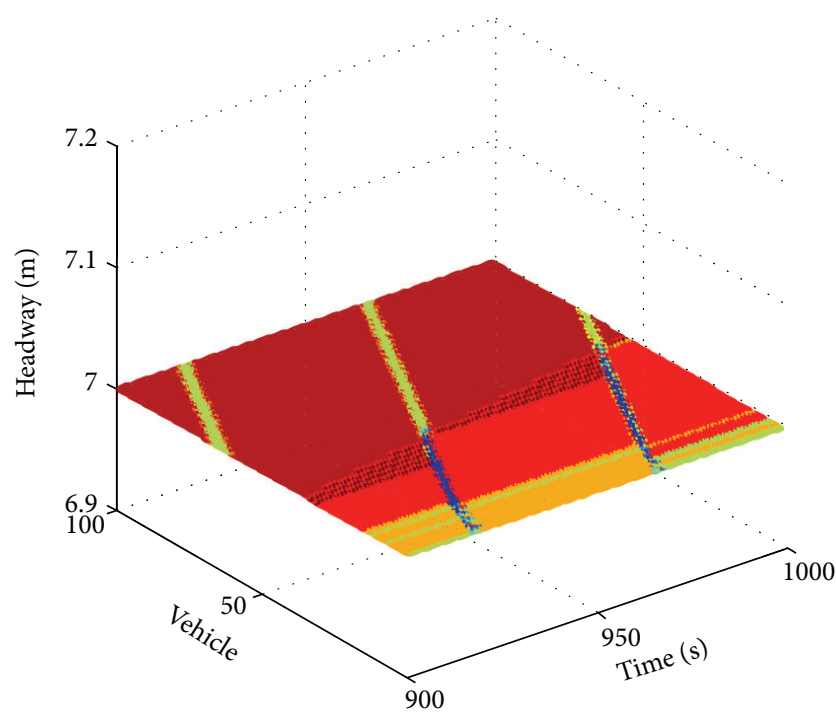

(b)

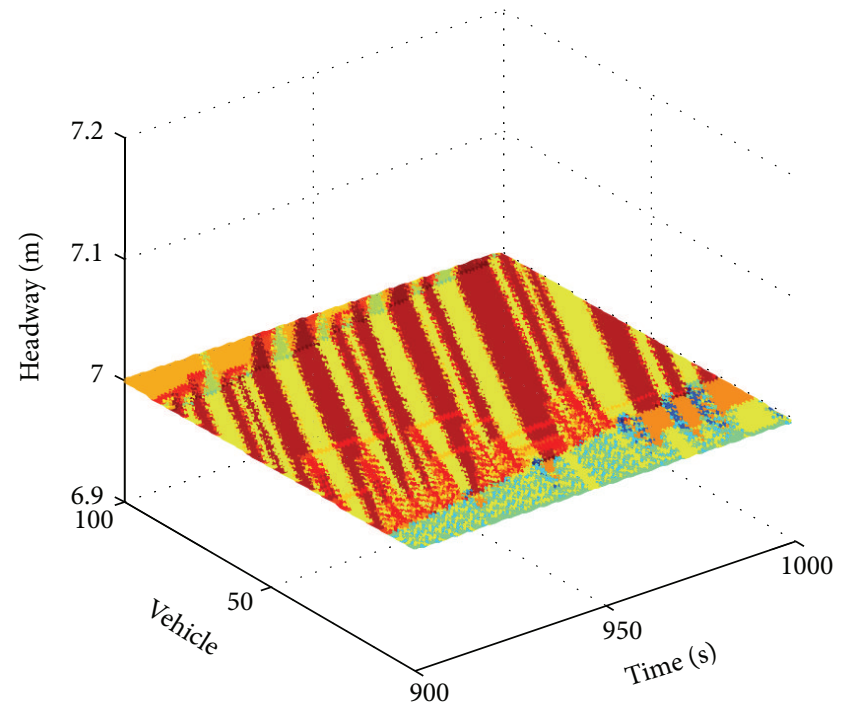

(d)

FIGURE 2: Space-time evolution of headway for lanel in (a) and lane2 in (b) when $p=0.8, q=0.2, \lambda_{1}=0.16$, and $\lambda_{2}=0.04$ and for lanel in (c) and lane2 in (d) when $p=0.6, q=0.4, \lambda_{1}=0.12$, and $\lambda_{2}=0.08$.

means the driver has no extra attention when considering lateral influence.

First add a disturbance on one lane as follows:

$$
\text { for lane1, } \begin{cases}\Delta x_{n}(0)=7-0.3 & 45<n<50 \\ \Delta x_{n}(0)=7 & \text { others; }\end{cases}
$$

for lane2, $\Delta x_{n}(0)=7 \quad 0<n \leq 100$.

Set $\alpha=2.85, p=0.8, q=0.2, \lambda_{1}=0.16$, and $\lambda_{2}=$ 0.04 for the first simulation test. Figure 2 shows the spacetime evolution of headway for lanel in (a) and lane2 in (b) from $900 \mathrm{~s}$ to $1000 \mathrm{~s}$. Then set $\alpha=2.85, p=0.6, q=0.4$, $\lambda_{1}=0.12$, and $\lambda_{2}=0.08$ for the next simulation. The results are shown in Figures 2(c) and 2(d). It is noticed that when the proportion of lateral influence increases from 0.2 to 0.4 , the density wave on lane 2 becomes heavier, while on lanel the perturbation decays.

Second, we consider both lanes have small disturbances, which are defined as follows:

$$
\begin{aligned}
& \text { for lane1, } \begin{cases}\Delta x_{n}(0)=7-0.1 & 45<n<50 \\
\Delta x_{n}(0)=7 & \text { others; }\end{cases} \\
& \text { for lane2, } \begin{cases}\Delta x_{n}(0)=7-0.3 & 45<n<50 \\
\Delta x_{n}(0)=7 & \text { others. }\end{cases}
\end{aligned}
$$

Let $\alpha=2.85, p=1, q=0, \lambda_{1}=0.2$, and $\lambda_{2}=0$, which cannot satisfy the stable condition in (10). Figure 3 


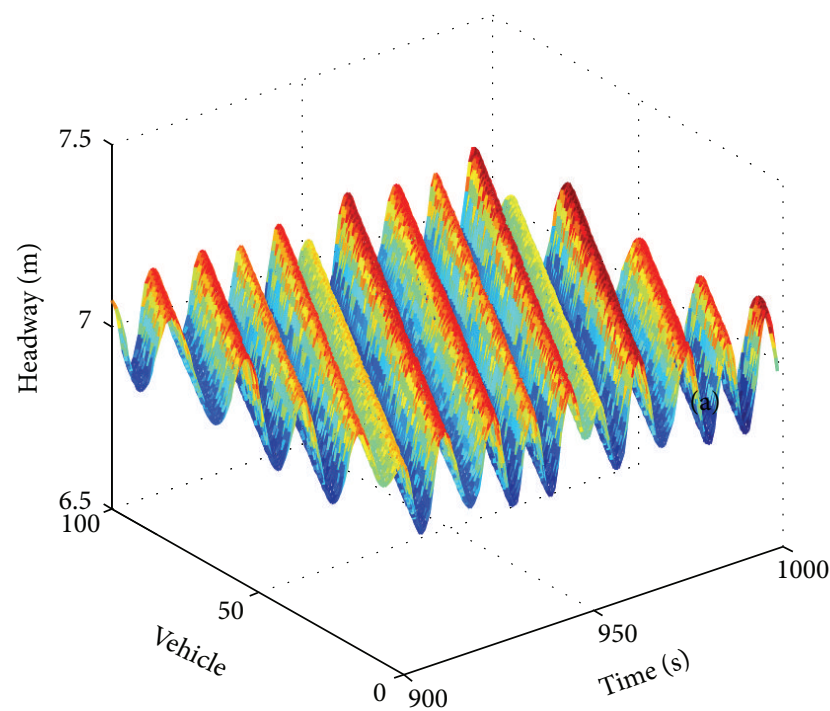

(a)

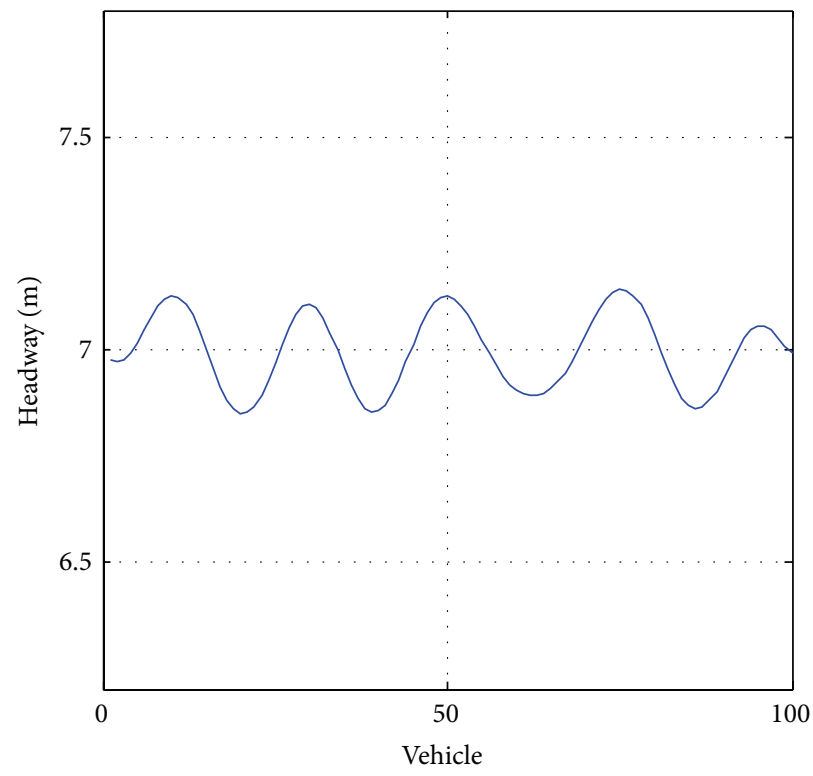

(c)

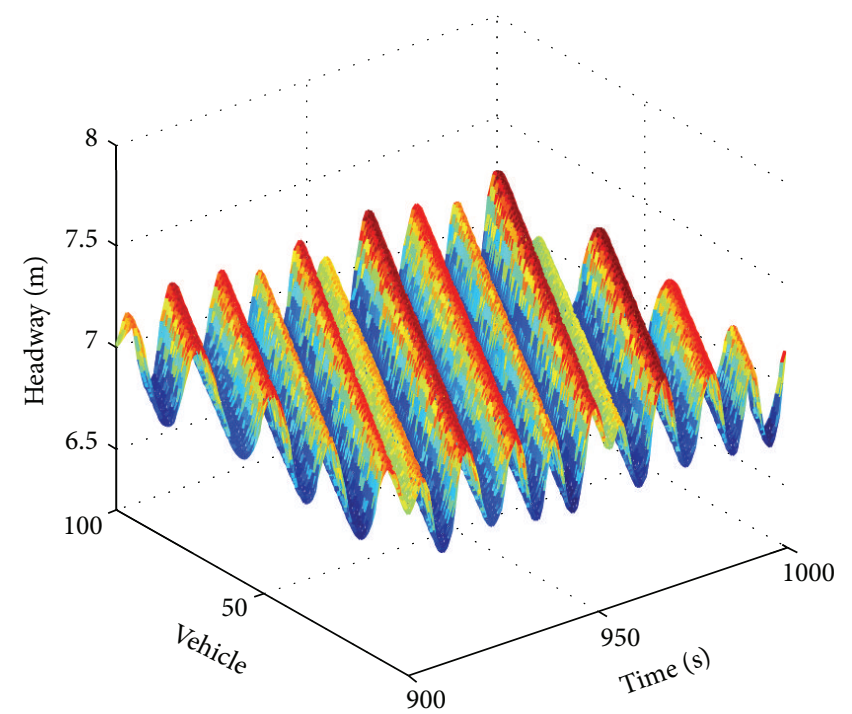

(b)

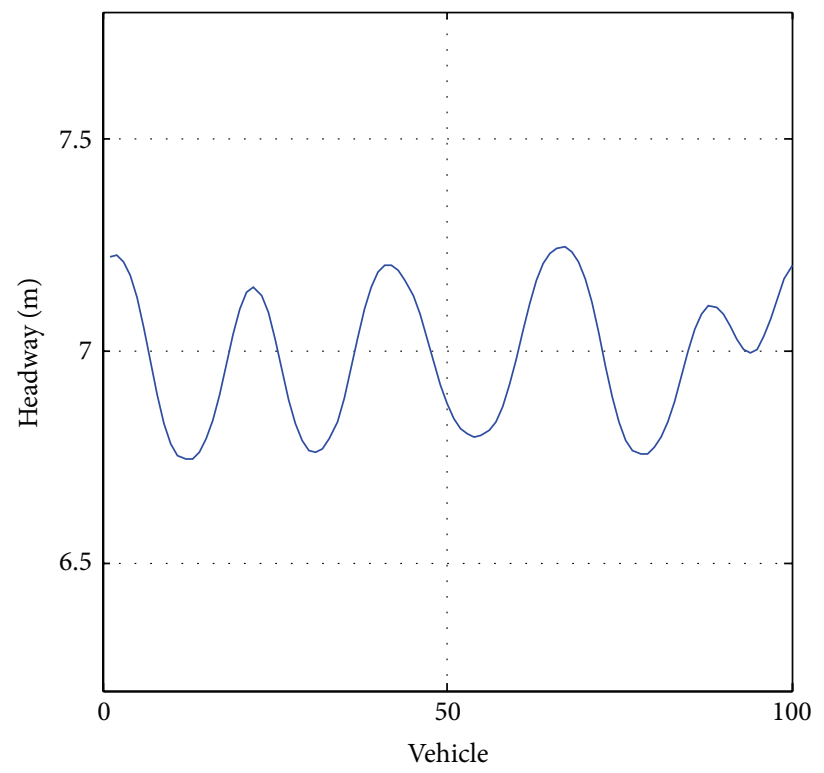

(d)

FIGURE 3: Space-time evolution of headway for lanel in (a) and lane2 in (b) and headway profile of traffic wave at $t=950 \mathrm{~s}$ for lanel in (c) and lane2 in (d). $\left(\alpha=2.85, p=1, q=0, \lambda_{1}=0.2\right.$, and $\left.\lambda_{2}=0\right)$.

shows the space-time evolution of headway for lanel in (a) and lane2 in (b) from $900 \mathrm{~s}$ to $1000 \mathrm{~s}$. The headway profiles of traffic wave at $t=950 \mathrm{~s}$ are in (c) for lanel and (d) for lane2. It is observed that the small perturbation propagates into traffic jam on both lanes. Furthermore, traffic jam is more serious in lane2 due to the larger initial headway perturbation.

Then suppose $\alpha=2.85, p=0.8, q=0.2, \lambda_{1}=0.16$, and $\lambda_{2}=0.04$. Figure 4 describes the space-time evolution of headway and the headway profiles of traffic wave at $t=950 \mathrm{~s}$ corresponding to Figure 3. The initial perturbation decays after sufficient time on both lanes. Thus the consideration of lateral impact from adjacent lane can suppress traffic jam.
The amplitude of traffic wave in lane 2 is larger just like in Figure 3.

Finally when $\alpha=2.2, p=0.8, q=0.2, \lambda_{1}=0.16$, and $\lambda_{2}=0.04$, the system is more unstable and serious kink-antikink waves are observed in Figure 5. Unlike Figures 3 or 4 , there is no significant difference between the two lanes, because all the vehicles get influenced heavily by lateral impact.

Then we exchange the disturbances of the two lanes, and simulations show similar results. In other words, the situation on lanel after exchange is like lane2 before exchange, the same for lane 2. By simulation, the theoretical analysis of MKdV solution can be described. 


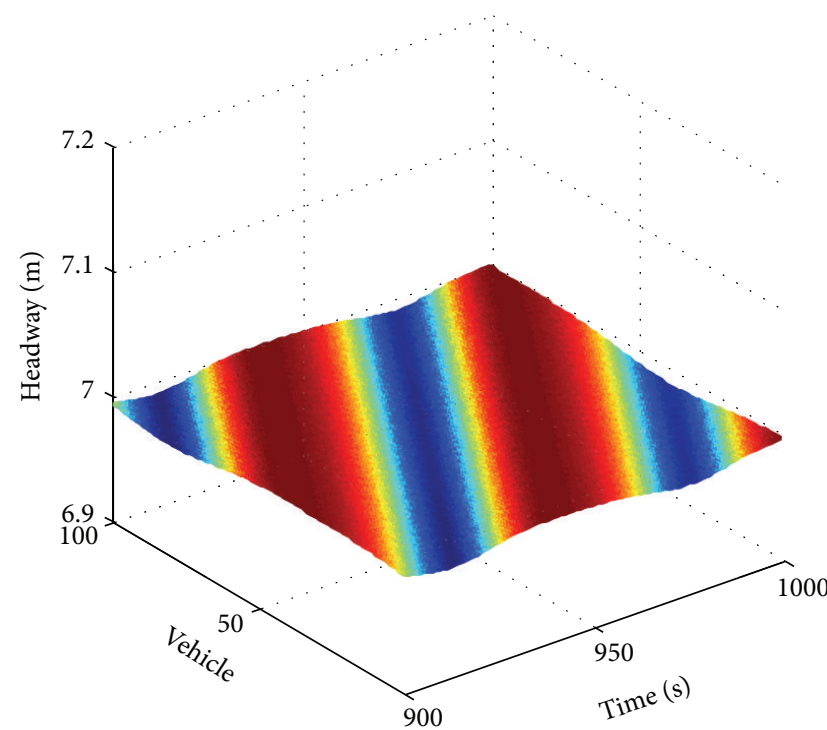

(a)

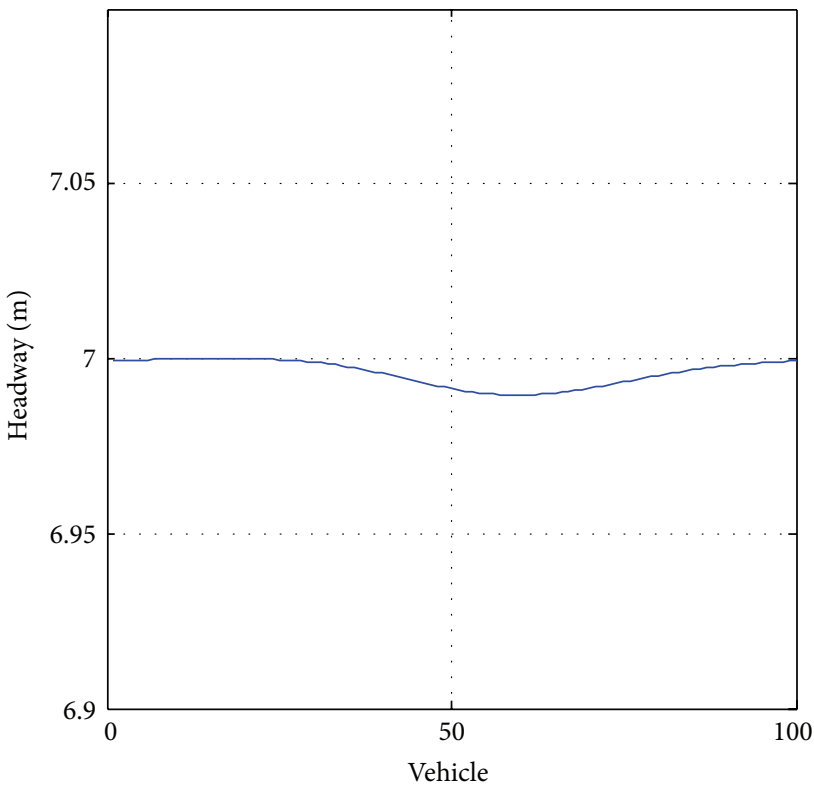

(c)

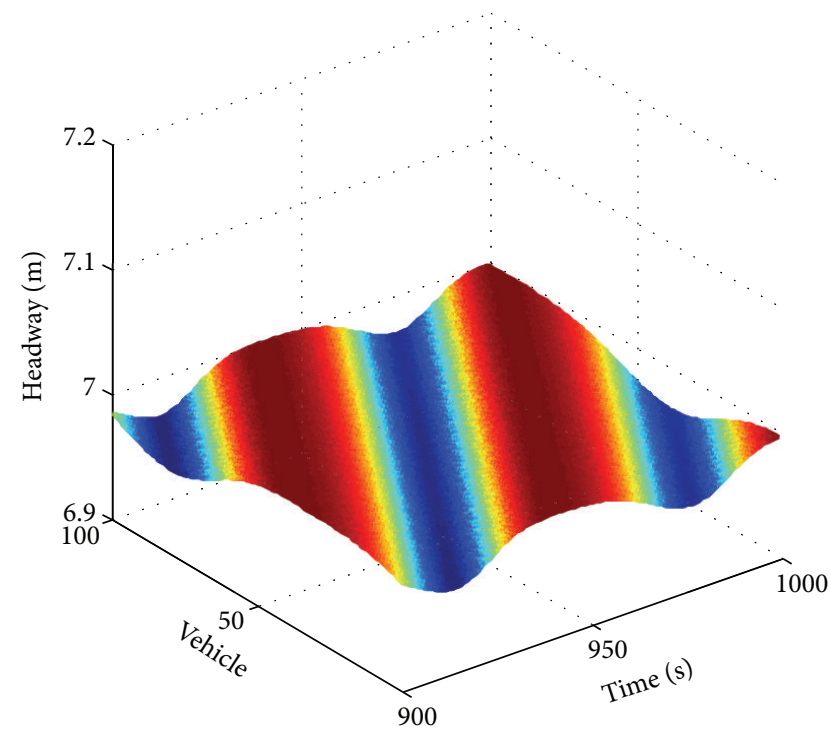

(b)

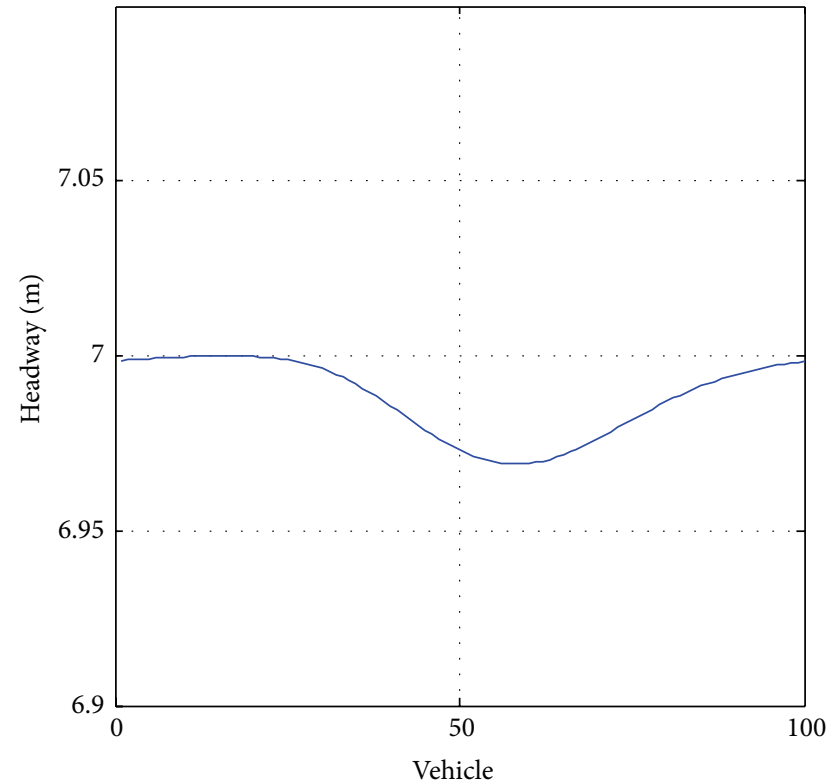

(d)

Figure 4: Space-time evolution of headway for lanel in (a) and lane2 in (b) and headway profile of traffic wave at $t=950 \mathrm{~s}$ for lanel in (c) and lane2 in (d). $\left(\alpha=2.85, p=0.8, q=0.2, \lambda_{1}=0.16\right.$, and $\left.\lambda_{2}=0.04\right)$.

\section{Conclusions}

In this paper, a new car-following model is proposed considering lateral influence from adjacent lane. Linear and nonlinear stability analyses are carried out, from which MKdV equation is obtained. Numerical simulations show that new model has good consistency with theoretical study and can suppress traffic jam wave. However, when $\alpha$ satisfied the unstable condition, both lanes will have serious traffic jam of same level despite different initial headway perturbation. In conclusion, even though lane changing does not happen, considering lateral impact has influence on car-following behavior indeed and can keep the vehicle system more stable.

\section{Conflict of Interests}

The authors declare that there is no conflict of interests regarding the publication of this paper.

\section{Acknowledgments}

The research in this paper was conducted as part of the project "The Occurrence and Evolution of Traffic Gridlock in 


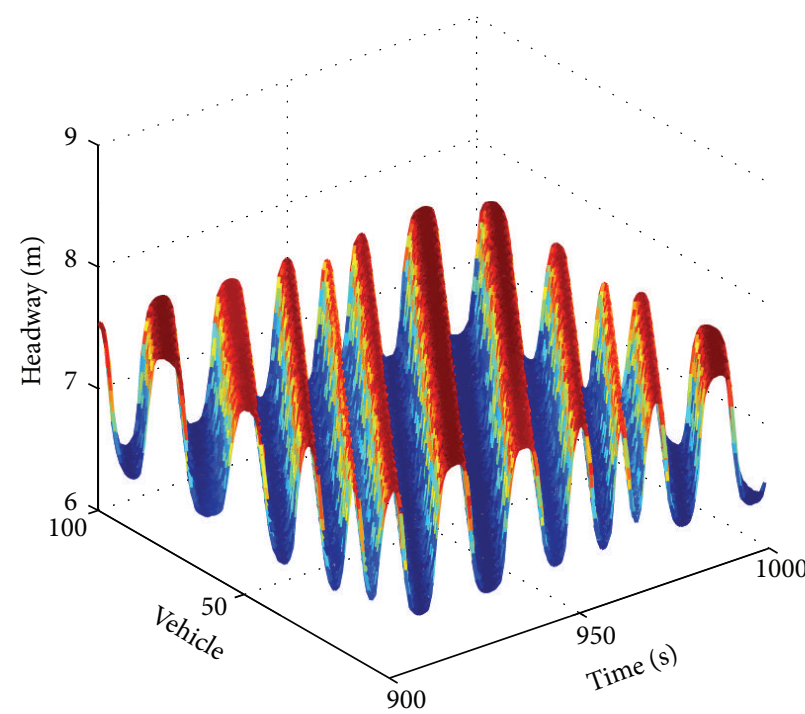

(a)

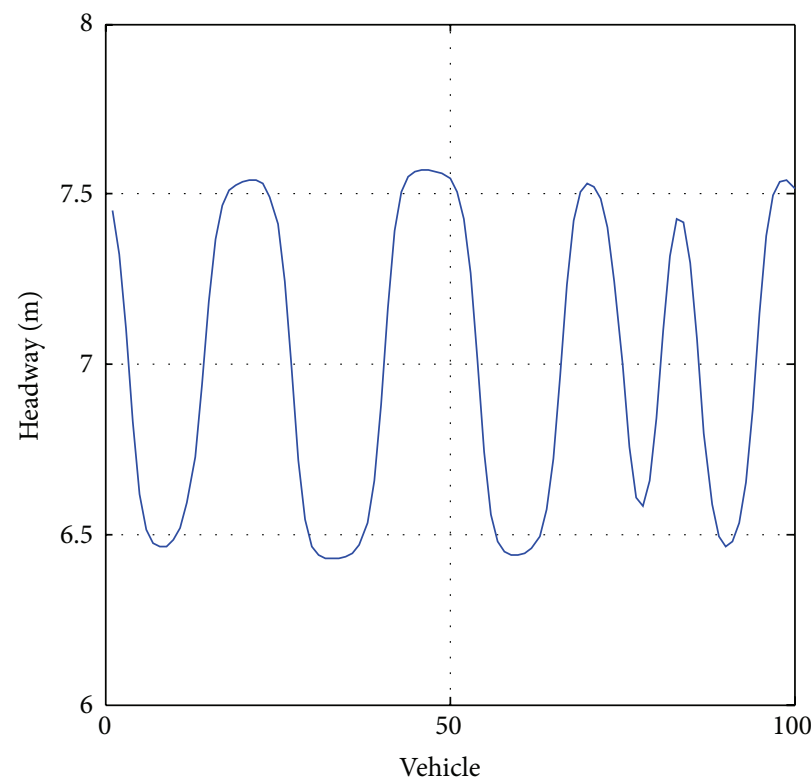

(c)

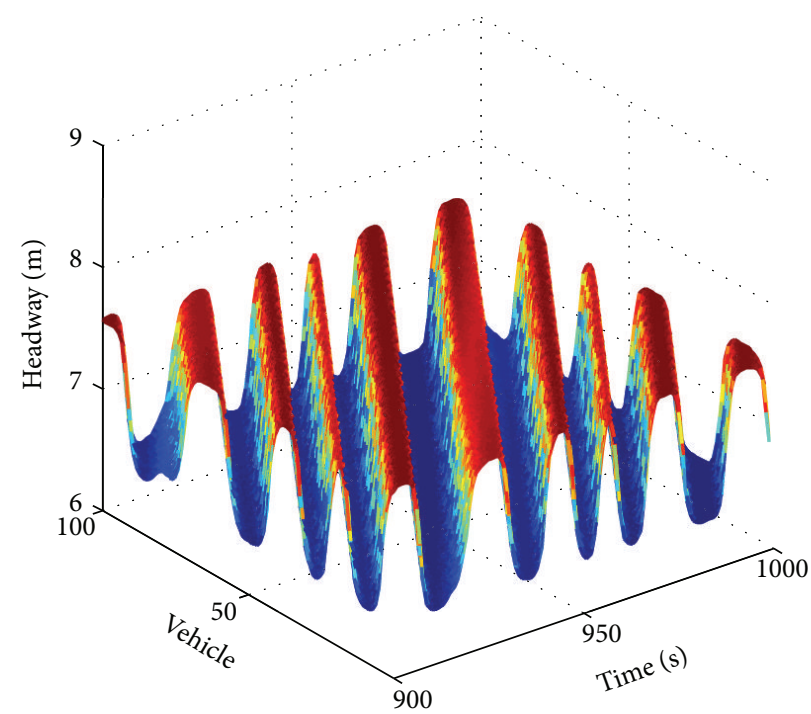

(b)

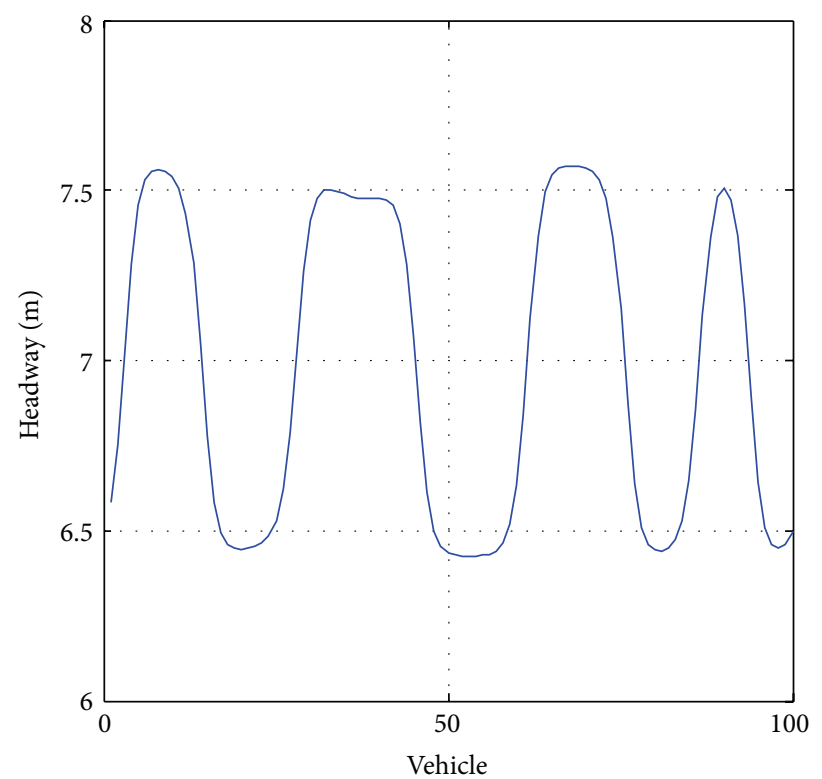

(d)

Figure 5: Space-time evolution of headway for lane1 in (a) and lane2 in (b) and headway profile of traffic wave at $t=950 \mathrm{~s}$ for lanel in (c) and lane2 in (d). $\left(\alpha=2.2, p=0.8, q=0.2, \lambda_{1}=0.16\right.$, and $\left.\lambda_{2}=0.04\right)$.

Mega-City under Storm Rain Conditions," which is funded by Beijing Natural Science Foundation (Project no. 20131100291). This research is also supported by the project "Research on the Traffic Environment Carrying Capacity and Feedback Gating Based Dynamic Traffic Control in Urban Network" which was funded by the China Postdoctoral Science Foundation with Grant no. 2013M540102.

\section{References}

[1] T. Nagatani, "The physics of traffic jams," Reports on Progress in Physics, vol. 65, no. 9, pp. 1331-1386, 2002.

[2] D. Helbing, "Traffic and related self-driven many-particle systems," Reviews of Modern Physics, vol. 73, p. 1067, 2001.
[3] M. Brackstone and M. McDonald, "Car-following: a historical review," Transportation Research F: Traffic Psychology and Behaviour, vol. 2, no. 4, pp. 181-196, 1999.

[4] J. Wu, M. Brackstone, and M. McDonald, "The validation of a microscopic simulation model: a methodological case study," Transportation Research Part C: Emerging Technologies, vol. 11, no. 6, pp. 463-479, 2003.

[5] M. Bando, K. Hasebe, A. Nakayama, A. Shibata, and Y. Sugiyama, "Dynamical model of traffic congestion and numerical simulation," Physical Review E, vol. 51, no. 2, pp. 1035-1042, 1995.

[6] M. Bando, K. Hasebe, K. Nakanishi, and A. Nakayama, "Analysis of optimal velocity model with explicit delay," Physical Review E, vol. 58, pp. 5429-5435, 1998. 
[7] D. Helbing and B. Tilch, "Generalized force model of traffic dynamics," Physical Review E, vol. 58, no. 1, pp. 133-138, 1998.

[8] M. Treiber, A. Hennecke, and D. Helbing, "Congested traffic states in empirical observations and microscopic simulations," Physical Review E, vol. 62, no. 2 B, pp. 1805-1824, 2000.

[9] R. Jiang, Q. Wu, and Z. Zhu, "Full velocity difference model for a car-following theory," Physical Review E-Statistical, Nonlinear, and Soft Matter Physics, vol. 64, no. 1, Article ID 017101, 4 pages, 2001.

[10] R. Jiang, S. Q. Wu, and Z. J. Zhu, "A new continuum model for traffic flow and numerical tests," Transportation Research Part B, vol. 36, no. 5, pp. 405-419, 2002.

[11] M. Treiber and A. Kesting, Traffic Flow Dynamics, Springer, Berlin, Germany, 2013.

[12] D. Helbing and M. Treiber, "Gas-kinetic-based traffic model explaining observed hysteretic phase transition," Physical Review Letters, vol. 81, no. 14, pp. 3042-3045, 1998.

[13] K. Konishi, H. Kokame, and K. Hirata, "Decentralized delayedfeedback control of an optimal velocity traffic model," European Physical Journal B, vol. 15, no. 4, pp. 715-722, 2000.

[14] L. C. Davis, "Modifications of the optimal velocity traffic model to include delay due to driver reaction time," Physica A: Statistical Mechanics and Its Applications, vol. 319, pp. 557-567, 2003.

[15] X. Zhao and Z. Gao, "A new car-following model: full velocity and acceleration difference model," The European Physical Journal B, vol. 47, pp. 145-150, 2005.

[16] T. Wang, Z. Gao Y, and X. Zhao M, "Multiple velocity difference model and its stability analysis," Acta Physica Sinica, vol. 55, pp. 634-640, 2006.

[17] G. H. Peng, X. H. Cai, C. Q. Liu, B. F. Cao, and M. X. Tuo, "Optimal velocity difference model for a car-following theory," Physics Letters, Section A: General, Atomic and Solid State Physics, vol. 375, no. 45, pp. 3973-3977, 2011.

[18] T. S. Komatsu and S.-I. Sasa, "Kink soliton characterizing traffic congestion," Physical Review E, vol. 52, no. 5, pp. 5574-5582, 1995.

[19] M. Muramatsu and T. Nagatani, "Soliton and kink jams in traffic flow with open boundaries," Physical Review E, vol. 60, pp. 180187, 1999.

[20] T. Nagatani, "Density waves in traffic flow," Physical Review E, vol. 61, pp. 3564-3570, 2000.

[21] T. Nagatani, "Modified KdV equation for jamming transition in the continuum models of traffic," Physica A: Statistical Mechanics and its Applications, vol. 261, no. 3-4, pp. 599-607, 1998.

[22] X. Yu, "Analysis of the stability and density waves for traffic flow," Chinese Physics, vol. 11, no. 11, pp. 1128-1134, 2002.

[23] H. X. Ge, R. J. Cheng, and Z. P. Li, "Two velocity difference model for a car following theory," Physica A: Statistical Mechanics and Its Applications, vol. 387, no. 21, pp. 5239-5245, 2008.

[24] H. X. Ge, R. J. Cheng, and S. Q. Dai, "KdV and kinkantikink solitons in car-following models," Physica A: Statistical Mechanics and Its Applications, vol. 357, no. 3-4, pp. 466-476, 2005.

[25] L. Yu, Z. K. Shi, and T. Li, "A new car-following model with two delays," Physics Letters A, vol. 378, no. 4, pp. 348-357, 2014.

[26] T. Nagatani, K. Nakanishi, and H. Emmerich, "Phase transition in a difference equation model of traffic flow," Journal of Physics A: Mathematical and General, vol. 31, no. 24, pp. 5431-5438, 1998.
[27] Z. H. Ou, S. Q. Dai, and L. Y. Dong, "Density waves in the full velocity difference model," Journal of Physics A, vol. 39, no. 6, pp. 1251-1263, 2006.

[28] T. Nagatani, "Jamming transitions and the modified Kortewegde Vries equation in a two-lane traffic flow," Physica A: Statistical Mechanics and Its Applications, vol. 265, no. 1-2, pp. 297-310, 1999.

[29] S. Jin, D. Wang, P. Tao, and P. Li, "Non-lane-based full velocity difference car following model," Physica A: Statistical Mechanics and its Applications, vol. 389, no. 21, pp. 4654-4662, 2010.

[30] H. X. Ge, X. P. Meng, J. Ma, and S. M. Lo, "An improved carfollowing model considering influence of other factors on traffic jam," Physics Letters A, vol. 377, no. 1-2, pp. 9-12, 2012. 


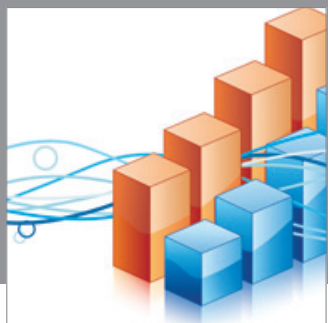

Advances in

Operations Research

mansans

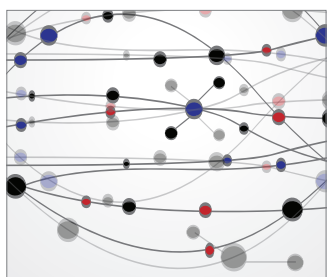

The Scientific World Journal
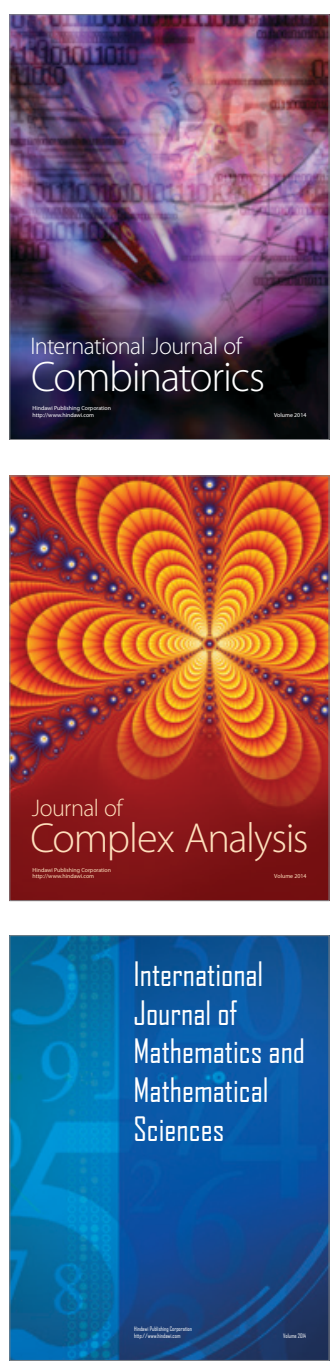
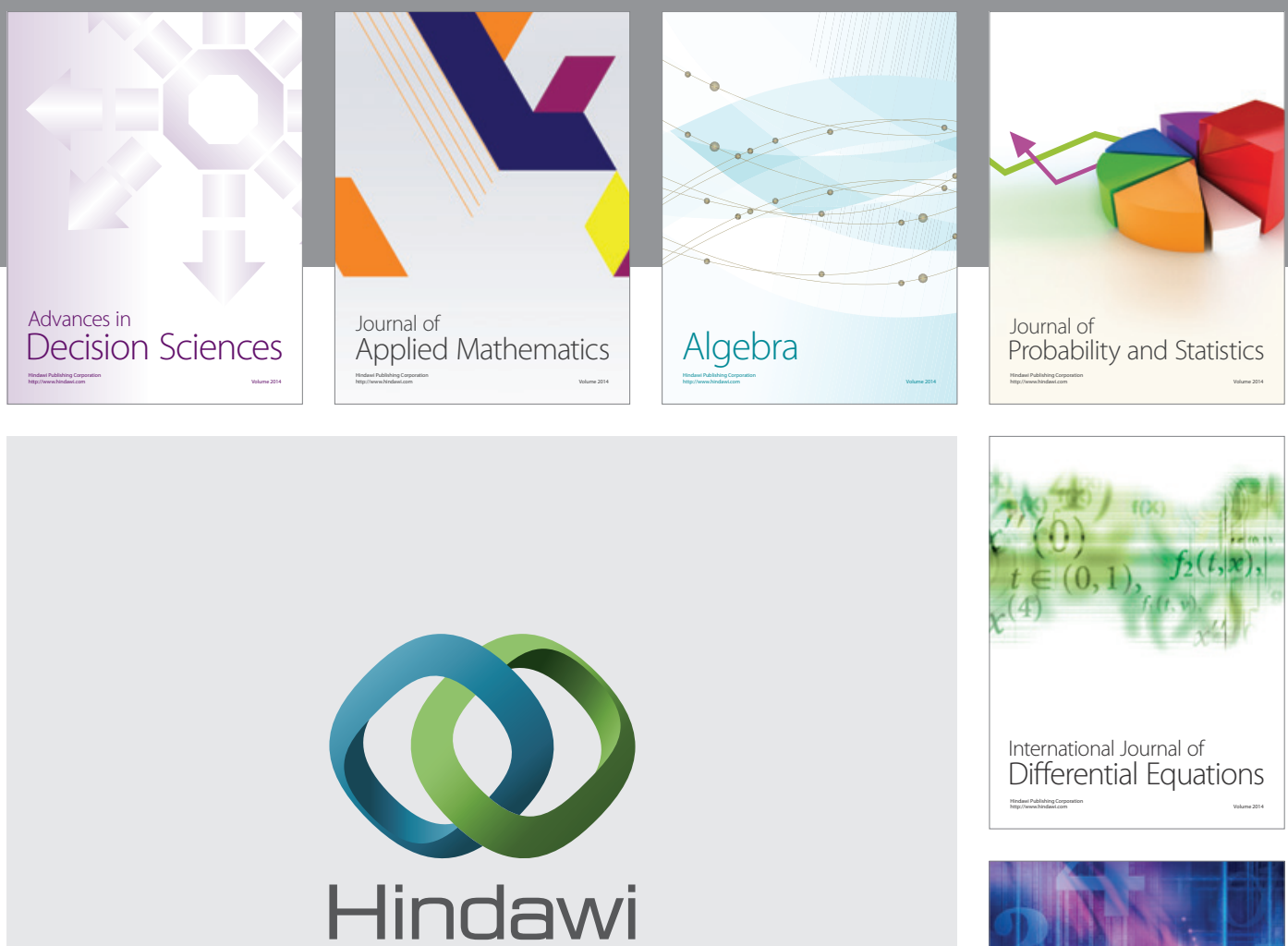

Submit your manuscripts at http://www.hindawi.com
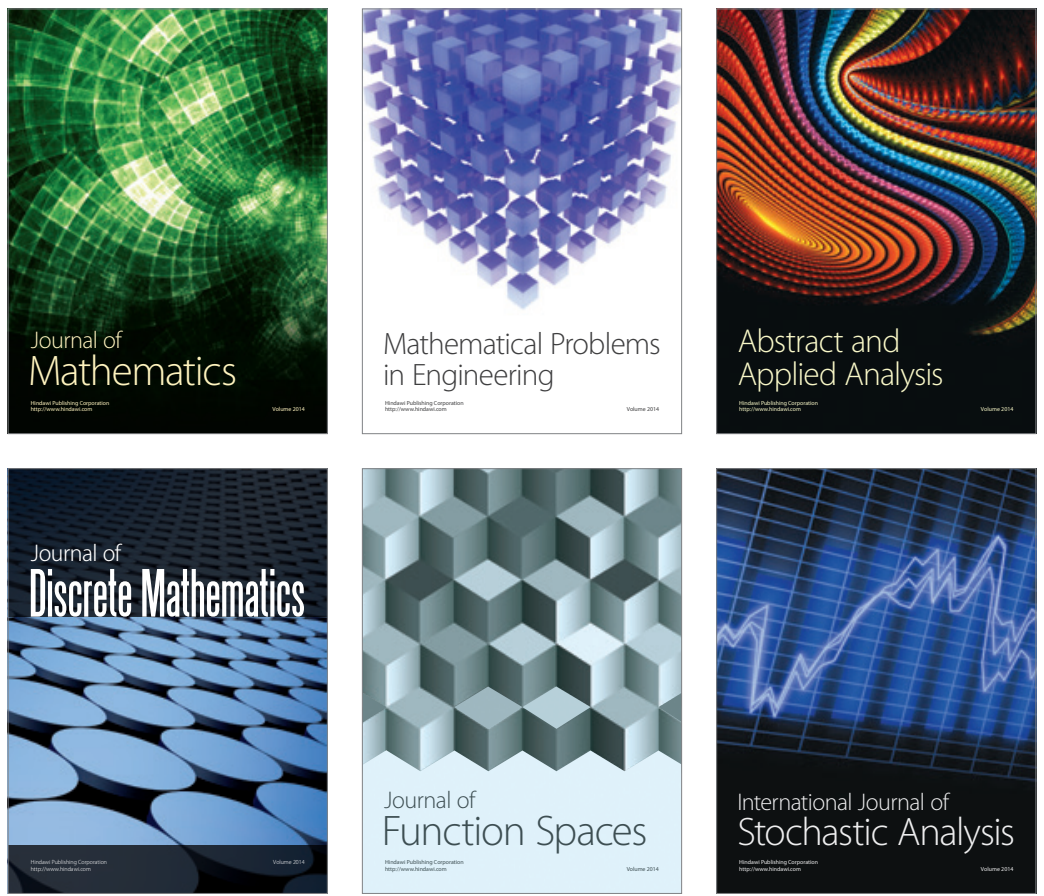

Journal of

Function Spaces

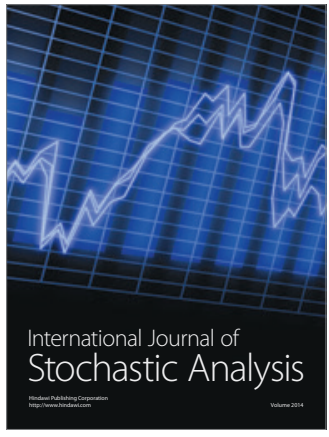

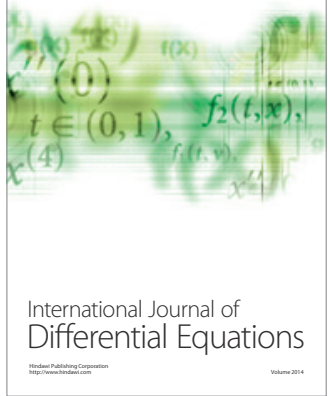
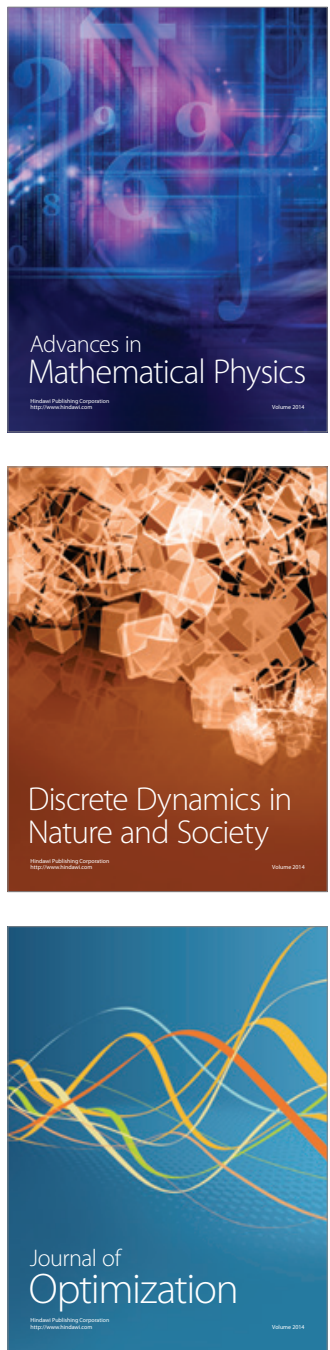